\title{
Intoxication accidentelle à l'arsenic par une pierre de collection
}

\author{
Accidental arsenic intoxication \\ with a collection stone
}

\section{Jean-Pierre GOULLÉ(1), Catherine KOLLY ${ }^{(2)}$, Christian LACROIX ${ }^{(1)}$, Anne DUQUENOY ${ }^{(3)}$, Gérard GAUSSIN ${ }^{(3)}$, Bernard LE LUYER ${ }^{(3)}$}

(1) Laboratoire de Pharmacocinétique et de Toxicologie Cliniques, Groupe Hospitalier, BP 24 - 76083 LE HAVRE Cedex - Tél : 0232733223 - Fax : 0232733238

(2) Service de Néonatologie, Centre Hospitalier Universitaire, 1, rue de Germont, 76031 ROUEN Cedex - Tél : 0232888990

(3) Département de Pédiatrie, Groupe Hospitalier -BP 24 - 76083 LE HAVRE Cedex Tél : 0232733630 - Fax : 0232733177

*Auteur à qui adresser la correspondance : Docteur Jean-Pierre GOULLÉ, Laboratoire de Pharmacocinétique et de Toxicologie Cliniques, Centre Hospitalier Jacques Monod - BP 24 - 76083 LE HAVRE

Tél : 0232733223 - Fax : 0232733238

(Reçu le 3 octobre 2000 ; accepté le 2 décembre 2000)

\section{$R \hat{E} S U M E$}

L'objectif de ce travail est de présenter un cas d'intoxication à l'arsenic par une pierre de collection et de proposer des mesures pour éviter ce type d'accident. L'observation que nous rapportons concerne un nourrisson de 17 mois qui joue avec les pierres de collection de son frère aîné. Celles-ci font partie d'un assortiment de pierres que l'on peut se procurer dans les magasins de souvenirs ou sur les marchés, sans qu'aucune mention ne soit faite de leur dangerosité éventuelle. Le jeune Flavian est surpris par sa mère avec des pierres dans la bouche. La mère le fait cracher et élimine le maximum de particules puis lui rince la cavité buccale à l'eau. Elle se rend aux Urgences Pédiatriques, l'enfant ne présente pas de symptomatologie clinique particulière. Les pierres, de couleur jaune vif, sont identifiées comme étant de l'orpiment ou sulfure naturel d'arsenic $\left(A s_{2} S_{3}\right)$. Le dosage de l'arsenic (As) est réalisé par spectrométrie d'absorption atomique électrothermique au four graphite, équipé d'un dispositif Zeeman pour la correction du bruit de fond (Spectra AA 800 avec GTA 100 Varian). La méthode des ajouts dosés que nous utilisons a été mise au point et validée au laboratoire selon les critères usuels. Le dosage spécifique de l'As inorganique, la seule forme d'As toxique, est effectué après extraction par

\section{SUMMARY}

The purpose of this paper is to present a case of arsenic intoxication with a collection stone and to propose guidelines aimed to avoid this type of accident. The case which we are reporting on is that of a 17 month old toddler who was playing with the stones' collection of his older brother. These stones formed part of an assortment which can be bought in a souvenir shop or in the markets without any mention of eventual dangers. The young Flavian was caught by his mother with some stones in his mouth. She made him spit them, eliminated as many particles as she could and rinsed his mouth with water. When she arrived to the paediatric emergency unit the child had no particular clinical symptoms. The stones, of a brilliant yellow colour, were identified as being orpiment or natural sulphur of arsenic $\left(A s_{2} S_{3}\right)$. Arsenic (As) level was measured by electrothermic atomic absorption spectrometry with a graphite oven equipped with a Zeeman device for the correction of background noise (Spectra AA 800 with GTA 100 Varian). The standard additions technique which we used was improved and validated in our laboratory according to the usual criteria. The specific dosage of inorganic As, the only toxic form of As, is done after extraction with chloroform. Inorganic urinary As sho- 
le chloroforme. L'As urinaire inorganique, montre une élévation très importante du rapport urinaire As/créatinine à 327 $\mu \mathrm{g} / \mathrm{g}$ (sujet exposé $<50 \mu \mathrm{g} / \mathrm{g}$ en fin de semaine de travail). Un contrôle de l'As sanguin et urinaire réalisé trois mois plus tard s'avère normal. Cette observation pose un réel problème de santé publique: minerais en vente libre, sans aucune mention, qui plus est destinés aux enfants alors qu'il s'agit de divers sels de métaux dont la toxicité peut être redoutable. Nous proposons un étiquetage adapté mentionnant la dangerosité éventuelle du minerai et l'emploi d'emballages inviolables pour les minéraux toxiques.

\section{MOTS-CLÉS}

Intoxication, arsenic, pierres de collection.

\section{Introduction}

Les pierres de collection, le plus souvent destinées aux enfants, sont en vente libre dans les magasins de souvenirs, sur les marchés ou dans des boutiques spécialisées en minéralogie. Elles sont très souvent présentées dans des boites transparentes sous forme d'assortiments colorés par lesquels les enfants sont très attirés. Cés boîtes contiennent les minéraux les plus divers sous différentes formes cristallines.

Si la majorité de ces minéraux ne présente pas de danger potentiel pour la santé en cas de contact ou d'ingestion accidentelle, il en va différemment pour d'autres, sels de métaux lourds en particulier. Les sels de métaux lourds fréquemment rencontrés sont des sels de plomb (galène ou $\mathrm{PbS}$ ) et des sels d'arsenic (orpiment ou $\mathrm{As}_{2} \mathrm{~S}_{3}$ ) sans qu'aucune mention ne soit faite de leur dangerosité éventuelle. L'observation que nous rapportons concerne un nourrisson de 17 mois qui joue avec les pierres de collection de son frère aîné. Nous proposons des mesures pour éviter ce type d'accident, mesures qui devraient être sans effet pour le commerce de ces minéraux.

\section{Observation}

Le jeune Flavian, âgé de 17 mois, est surpris par sa mère avec des pierres de collection dans la bouche. La mère le fait cracher pour éliminer le maximum de particules et lui rince la bouche à l'eau. Elle se présente affolée aux urgences pédiatriques. Les pierres de couleur jaune vif sont identifiées comme étant de l'orpiment ou sulfure naturel d'arsenic $\left(\mathrm{As}_{2} \mathrm{~S}_{3}\right)$. L'enfant ne présente pas de symptomatologie clinique particulière. On note l'absence de vomissement et de douleur abdominale. Le reste de l'examen clinique et le bilan biologique sont normaux. Un lavage gastrique est réalisé, Flavian est ensuite traité par charbon activé puis perfu- wed a very important elevation of the ratio urinary As/creatinine at $327 \mu \mathrm{g} / \mathrm{g}$ (exposed subject $<50 \mu \mathrm{g} / \mathrm{g}$ at the end of a working week). A control of blood and urinary as done afterwards was normal. This observation puts in evidence a real problem of public health. Minerals containing different mineral salts, some of which might be of fearful toxicity, are freely sold without any indications of danger and what is even worse they are intended for children. We suggest minerals should be provided with a tag indicating the eventual danger of the mineral and the use of sealed packages for the toxic minerals.

\section{KEY-WORDS}

intoxication, arsenic, stone collections.

sé. La radiographie de l'abdomen sans préparation permet de mettre en évidence plus d'une dizaine de minuscules fragments métalliques (figure 1). Un dosage d'arsenic urinaire est effectué ; il est très élevé : $327 \mu \mathrm{g} / \mathrm{g}$. L'enfant reste asymptomatique et l'évolution est favo-

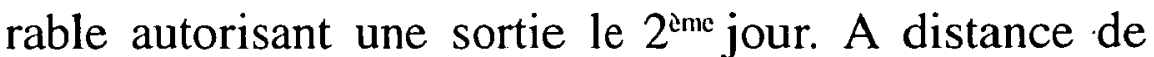
l'intoxication, des contrôles de l'arsenic sanguin et urinaire sont réalisés ; ils sont normaux.

\section{Matériel et méthode : Arsenic urinaire}

Le dosage est réalisé par spectrophotométrie d'absorption atomique électrothermique avec correction Zeeman selon la méthode des ajouts dosés mise au point et validée au laboratoire selon les critères usuels. La technique décrite permet de mesurer l'arsenic inorganique et ses métabolites : les acides monométhyl et diméthylarsénique après élimination de l'arsénobétaïne par extraction chloroformique (1).

\section{Réactifs}

Tous les réactifs sont au minimum de qualité analytique : eau stérile Baxter (réf. F 7114), étalon d'arsenic à $1 \mathrm{~g} / \mathrm{l}$ Merck (réf. 19173), nitrate de nickel Merck (réf. 6721), iodure de potassium Prolabo (réf. 26846 235), chloroforme Carlo Erba (réf. 43860), acide chlorhydrique suprapur Merck (réf. 1.00318), acide nitrique suprapur Merck (réf. 1.00441).

\section{Préparation de l'échantillon}

A $800 \mu \mathrm{l}$ d'urines on ajoute $1200 \mu \mathrm{l}$ d'acide chlorhydrique concentré et $300 \mu \mathrm{l}$ d'iodure de potassium $3 \mathrm{M}$. Un blanc et des ajouts dosés à un pool d'urines $(\mathrm{x}, \mathrm{x}+50 \mu \mathrm{g} / \mathrm{l}$ et $\mathrm{x}+100 \mu \mathrm{g} / \mathrm{l} \mathrm{d}$ 'As) sont traités dans les mêmes conditions. Après agitation, laisser reposer 
Annales de Toxicologie Analytique, vol. XIII, $n^{\circ}$ 1, 2001

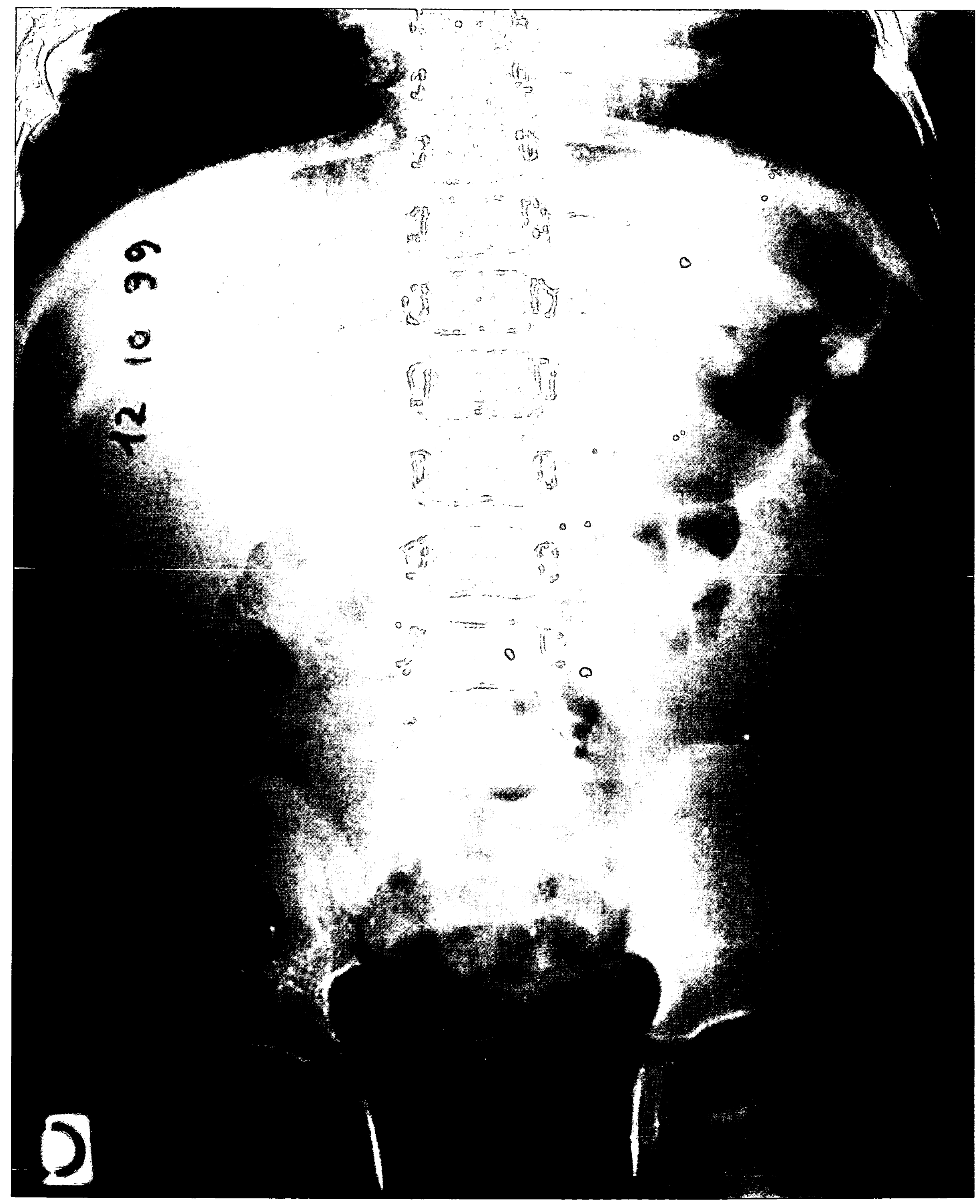

Figure 1 : Radiographie de l'abdomen sans préparation. 
$10 \mathrm{~min}$. Une extraction est réalisée par $1500 \mu \mathrm{l}$ de chloroforme et agitation pendant $5 \mathrm{~min}$, puis centrifugation. La phase aqueuse est éliminée par aspiration. Dans un autre tube à $800 \mu \mathrm{l}$ d'eau on ajoute $100 \mu \mathrm{l}$ de la phase chloroformique précédente. Chaque tube est agité 5 min puis centrifugé. $400 \mu \mathrm{l}$ de la phase aqueuse sont recueillis et placés sur le passeur d'échantillons. $400 \mu \mathrm{l}$ de modificateur de matrice (nitrate de nickel à $1 \%$ dans $\mathrm{HNO}_{3} 0,02 \mathrm{~N}$ ) sont disposés sur le passeur d'échantillon à l'emplacement prévu à cet effet.

\section{Appareillage}

Les analyses sont réalisées sur un spectromètre d'absorption atomique Spectra AA 800 Varian (Orsay, France), équipé d'un four GTA 100 , relié à un ordinateur Deskpro XE 466 Compaq et à une imprimante Desk Jet 520 Hewlett Packard (Les Ulis, France). La programmation de l'automate comportant en particulier outre les différents réglages habituels, l'adjonction du modificateur de matrice et les températures de séchage de minéralisation et d'atomisation du four, est reportée tableau I.

\section{Résultats}

\section{Validation de la méthode}

La limite de détection est de $1,2 \mu \mathrm{g} / \mathrm{l}$ (rapport signal/bruit de fond $=3$ ). La répétabilité à la concentration de $106 \mu \mathrm{g} / \mathrm{l}$ s'établit à 3,8\% $(\mathrm{n}=20)$. La linéarité est comprise entre 20 et $180 \mu \mathrm{g} / \mathrm{l}$. La sensibilité exprimée par la masse caractéristique donnant un signal d'absorption de $1 \%$ (soit 0,0044 unités d'absorbance) est de $21 \mathrm{pg}$. La récupération d'ajouts dosés d'As minéral atteint $97 \%$ à la concentration de $100 \mu \mathrm{g} / \mathrm{l}$ et $95 \%$ à $200 \mu \mathrm{g} / \mathrm{l}$ après dilution automatique de l'échantillon puisque cette concentration est supérieure à la linéarité de la méthode : $180 \mu \mathrm{g} / \mathrm{l}$.

\section{Arsenic urinaire du jeune Flavian}

L'arsenic urinaire après élimination de l'arsénobétaïne par le chloroforme (essentiellement les acides monométhyl et diméthylarséniques) de l'enfant est rapportée à la créatinine : As/créatinine $=327 \mu \mathrm{g} / \mathrm{g}$. Les valeurs usuelles chez un sujet non exposé sont $<20 \mu \mathrm{g} / \mathrm{g}$ de créatinine. Chez un sujet exposé la concentration urinaire doit rester $<50 \mu \mathrm{g} / \mathrm{g}$ de créatinine en fin de semaine d'exposition.

\section{Discussion}

Cette observation est intéressante à plusieurs titres :

- Elle montre que la radiographie de l'abdomen sans
Tableau I : Conditions opératoires pour le dosage de l'arsenic urinaire inorganique.

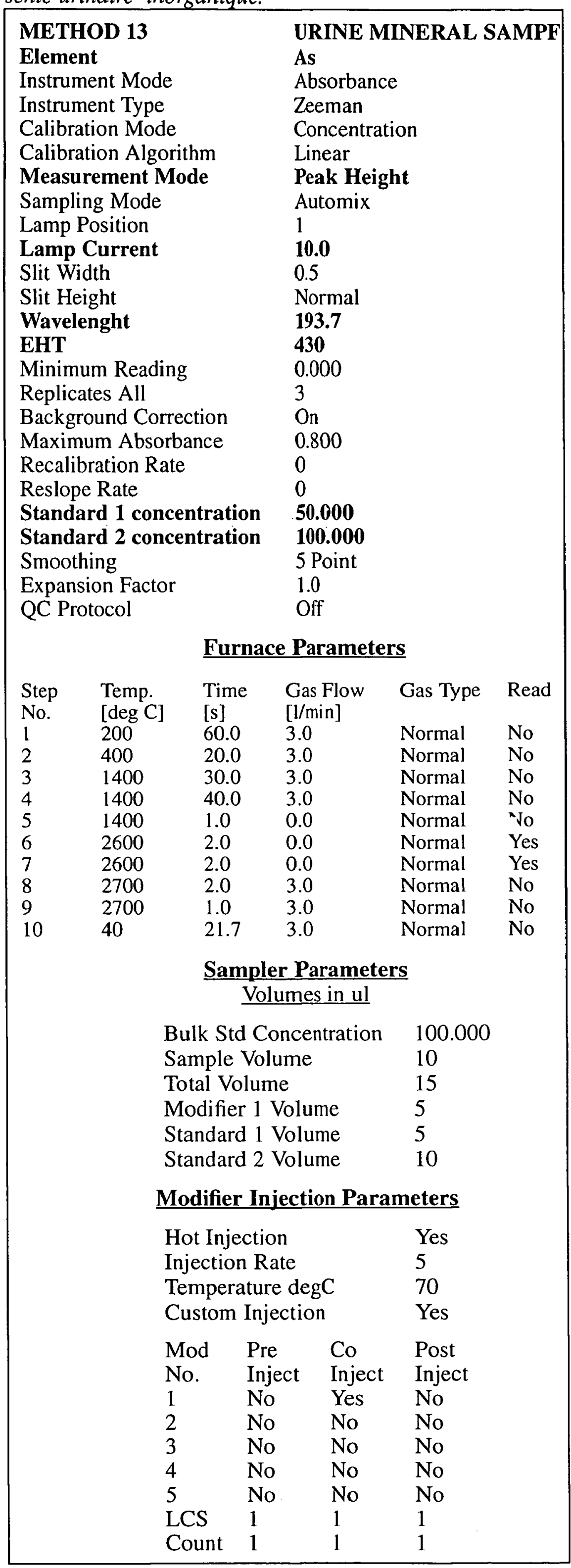


préparation est un examen de choix dans toutes les intoxications par les métaux lourds ou/et leurs sels, en raison de leur opacité aux rayons $X$ qu'il s'agisse de l'arsenic, du thallium, du plomb ou du mercure (réf. 2 à 4), cette liste n'étant pas limitative.

- Elle met en lumière l'utilité de documenter les cas d'intoxication et/ou d'exposition par l'analyse toxicologique. Même s'il n'est pas utile de pratiquer à chaque fois ces examens, des prélèvements doivent au moins être réalisés à titre conservatoire ; ceux-ci pourront être analysés en fonction de l'évolution clinique du malade et/ou du contexte particulier.

- Elle pose un réel problème de santé publique ; cet orpiment ou sulfure naturel d'arsenic est d'une couleur jaune vif, très attirante surtout pour les plus jeunes enfants, et ces assortiments sont en effet essentiellement destinés aux enfants. L'évolution de l'intoxication aurait pu être beaucoup plus grave, même si l' $\mathrm{As}_{2} \mathrm{~S}_{3}$ est beaucoup moins toxique, en raison d'une plus faible solubilité, par rapport à d'autres sels : acide arsénique, anhydride arsénieux $\left(\mathrm{As}_{2} \mathrm{O}_{3}\right)$ en particulier.

\section{Références}

1. A. Le Bouil et al., Dosage de l'arsenic urinaire après séparation de l'arsénobétaïne. Toxicorama, 1997 ; 9 : 171-176.

2. J.R. Gray, A. Khalil, J.C. Prior. Acute arsenic toxicity on opaque poison. Can. Assoc. Radiol. J., 1989 ; 40 : 226227.

3. M.K. Kulshrestha. Lead poisoning diagnosed by abdominal $\mathrm{X}$ rays. J. Toxicol. Clin. Toxicol., 1996 ; 34 : 107108.

4. P. Sanz Gallén, S. Nogué, P. Luburich, P. Munne, H. Lacalle, R. Reig. Hallazgos radiológicos en intoxicaciones por metales. Rev. Esp. Enferm. Apar. Dig., 1992 ; $81: 205-208$.

5. J.P. Goullé. Métaux. In : Toxicologie et pharmacologie médicolégales. Coordinateur : P. Kintz. Collection Option BIO, Elsevier, 1998 : 189-232.
Classiquement, une quantité d' $\mathrm{As}_{2} \mathrm{O}_{3}$ comprise entre 200 et $300 \mathrm{mg}$ pour un sujet adulte peut avoir une conséquence mortelle (5). En ce qui concerne $\mathrm{As}_{2} \mathrm{~S}_{3}$, ce sel est peu soluble en milieu acide, et très soluble en milieu alcalin. Le lavage gastrique très précoce réalisé chez le jeune Flavian, a vraisemblablement été déterminant. Les rares particules qui n'ont pu être éliminées par cette technique et qui sont visibles à la radiographie ont été partiellement solubilisées dans le milieu intestinal alcalin et sont à l'origine de l'élévation de l'As minéral urinaire.

\section{Conclusion}

Nous suggérons pour éviter la multiplication de ce type d'accident un étiquetage adapté mentionnant la dangerosité éventuelle des minéraux toxiques destinés aux enfants. Nous proposons que ces minéraux soient conditionnés dans des emballages transparents inviolables. 